\title{
Strategies to Control the Spectral Properties of Au-Ni Thin Films
}

David J McPherson ${ }^{\mathrm{a}}$, Supitcha Supansomboon ${ }^{\mathrm{a}}$, Benjamin Zwan ${ }^{\mathrm{b}}$, Vicki J Keast ${ }^{\mathrm{b}}$, David L Cortie ${ }^{\mathrm{c}, \mathrm{d}}$, Angus Gentle $^{\mathrm{a}}$, Annette Dowd ${ }^{\mathrm{a}}$ and Michael B Cortie ${ }^{\mathrm{a} *}$

${ }^{a}$ Institute for Nanoscale Technology, University of Technology Sydney, PO Box 123, Broadway NSW 2007, Australia

${ }^{b}$ School of Mathematical and Physical Sciences, University of Newcastle, Callaghan NSW 2308, Australia

'Institute for Superconducting and Electronic Materials, University of Wollongong, Wollongong, NSW 2522, Australia

${ }^{d}$ Australian Nuclear Science and Technology Organisation, Lucas Heights, NSW 2234, Australia

*Corresponding author: Tel.: +612 95142208

E-mail address: michael.cortie@uts.edu.au

Keywords: physical vapor deposition, optical properties, multilayers, ab initio calculation, gold-nickel

\begin{abstract}
Gold and nickel have quite different dielectric functions. Here we use a combination of calculation and sample manufacture to assess two strategies by which thin films of these elements can be produced with a controlled range of far-field optical properties. In the first approach, control can be achieved by manipulating the density of states of metastable solid solutions, which in turn controls the dielectric function. In the second approach the optical properties of the films is controlled by varying the geometry of stacks fabricated from the constituent elements. We show that the two approaches can produce equivalent results so both are viable options in practice. Modeling is used to reveal how the structure controls the optical properties and to map out the possible color gamut. Predictions are tested with thin film samples fabricated by magnetron sputtering.
\end{abstract}




\section{Introduction}

The yellow color of bulk gold is the consequence of the element's unusual dielectric function . Specifically, there is a well-defined band edge at about $2.4 \mathrm{eV}$ (equivalent to green light, at a vacuum wavelength of about $515 \mathrm{~nm}$ ). This band edge corresponds to the minimum photon energy capable of exciting electronic interband transitions from occupied to unoccupied states. Impinging photons of greater energy (i.e. shorter wavelength) are readily absorbed by the metal whereas photons of lesser energy will normally be scattered (reflected). Therefore, the reflectance of Au surfaces rises sharply as the wavelength of light is increased beyond the band edge. This results in the surface reflecting red, orange and yellow colors much more strongly than the blue to green colors, giving gold its characteristic color. In contrast, the band edge of $\mathrm{Ag}$ and most other metals lies in the UV, and hence they appear colorless (silver) to the human eye.

Here we analyze and compare two different microstructural strategies by which $\mathrm{Ni}$ can be used to modify the spectral properties of thin films of $\mathrm{Au}$. The strategies are generic and, in principle, certain other elements, such as Fe, could be substituted for the Ni.

In the first approach we modify the electronic density-of-states (DOS) of the Au by adding Ni as an alloying element. This 'bleaches' (whitens) the color by modifying the location of the band edge and strength of the interband transitions . Actually, while mutually soluble above $810^{\circ} \mathrm{C}$, the equilibrium solubility of $\mathrm{Ni}$ in $\mathrm{Au}$, and vice versa, is negligible at room temperature because the binary phase diagram is characterized by a complete miscibility gap at room temperature, Fig.1. Therefore, at first sight, bleaching of thin films of Au by modification of its band structure with $\mathrm{Ni}$ should not be possible at room temperature. Nevertheless, bleaching by this means is still feasible because metastable solutions of Ni in Au can be prepared, for example by annealing an alloy sample above $1000 \mathrm{~K}$ and then quenching it to room temperature, or by codepositing $\mathrm{Ni}$ and Au onto a cold substrate using physical vapor deposition.

There have been only a few studies on the optical properties of such metastable Au-Ni alloys or on those of the somewhat analogous Au-Fe system . In general, the presence of transition metal impurities such as $\mathrm{Ni}$ are expected to generate a virtual bound state (VBS) within the conduction band of the Au with the width of the VBS increasing as the impurity content increased. This would cause enhanced scattering of 
conduction band electrons, i.e. a reduction in electrical conductivity, particularly at energies below the band edge . This is usually correlated with an increase in optical 'loss' and decreased reflectance. However, addition of Ni to Au concurrently causes a reduction of the interband absorption at energies above the band edge, in proportion to the quantity of $\mathrm{Ni}$ added. The net result of these two opposing tendencies is that the reflectance at wavelengths above the band edge (i.e. towards the infrared) is reduced (due to the reduction in conductivity) whilst the reflectance at wavelengths below the band edge (ie. towards the ultraviolet) is increased. The overall effect is to de-saturate the color and, in the CIE L*a*b* color system, attenuate the luminance (brightness) of the alloy's surface.

The second approach to controlling the spectral properties exploits the immiscibility of Au and $\mathrm{Ni}$ at room temperature. In this case the far-field spectral properties are derived from a nanoscale mixture of the pure elements. Provided the size scale of the nanostructure is well below the incident wavelength, light can penetrate through it and interact with buried features. The result is that the far-field optical properties of the alloy are a hybrid combination of those of the individual phases. In a sense, the heterogeneous microstructure may be thought of as a meta-material whose effective dielectric properties are determined by the volume fraction and morphology of the Au and Ni regions and, possibly, by the amount of Ni remaining in the metastable solid solution of the Au-rich phase, and vice versa.

\section{Methodology Section}

\subsection{Calculations using Density Functional Theory}

In this study, calculations of the DOS for a number of different nickel concentrations were made using WIEN2k, an all-electron, linear augmented plane-wave density functional theory (DFT) code . The complex dielectric function, $\varepsilon=\varepsilon_{1}+i \varepsilon_{2}$, was calculated using the OPTIC routine within WIEN2k. These calculations are based on the random phase approximation and neglect local field effects . The dielectric function was then used to obtain a theoretical reflectivity spectrum. The structures were generated by replacing atoms in the gold fcc structure with increasing numbers of Ni atoms. Note that WIEN2k does not simulate a random solid 
solution, and the Ni atoms in our simulations are on a periodic lattice. However, different configurations of $\mathrm{Ni}$ atoms were tested for $\mathrm{Au}_{0.875} \mathrm{Ni}_{0.125}$ and the effect on the optical response was found to be negligible. Similarly, relaxation of the lattice parameter of this alloy gave values to within $1 \%$ of that of pure Au and had a negligible effect on the optical response.

\subsection{Optical property calculations}

The properties of thin film stacks were modeled using OpenFilters . This freely-available software calculates the optical properties of a stack of thin films using analytical expressions derived using the characteristic matrix approach. Colors are estimated by applying the CIE color space model, a standard illuminant (CIED65 white light) and a standardized observer (CIE-1964) to the calculated reflectance spectra. The published dielectric functions of bulk, annealed gold and nickel were used. The simulations included $1 \mathrm{~mm}$ of fused $\mathrm{SiO}_{2}$ glass as a substrate.

\subsection{Preparation of thin films}

The experimental samples for our study were deposited onto clear glass slides (Knittel Glaser). These had been cleaned by sonication in a sequence of acetone, ethanol and then water for 10 minutes at each step, rinsed with ultrapure water and immediately dried with $\mathrm{N}_{2}$. Film fabrication was carried out using dual magnetron sputtering from $\mathrm{Au}$ and $\mathrm{Ni}$ targets of $50 \mathrm{~mm}$ diameter using independent control of electrical power (typically $62 \mathrm{~W}$ for the $\mathrm{Au}$, with the power on the Ni target varied between 26 and $60 \mathrm{~W}$ ). Deposition rates were calibrated using a quartz crystal sensor and were of the order of 0.1 to $0.3 \mathrm{~nm} . \mathrm{s}^{-1}$ for the alloy. The substrate was at room temperature, the base pressure was about $1.3 \times 10^{-4} \mathrm{~Pa}$ and the operating pressure in the chamber in the range 0.24 to $0.28 \mathrm{~Pa}$ of Ar. A rotating stage was used to provide a uniform coverage. In the case of multilayer samples, the first and last layers were $\mathrm{Au}$, with the Au top layer intended to prevent oxidation of the $\mathrm{Ni}$ intermediate layer. 


\subsection{Characterization of thin films}

Glancing angle $\mathrm{X}$ ray diffraction (XRD) and $\mathrm{X}$ ray reflectometry (XRR) was carried out on a PANanalytical X'Pert PRO diffractometer with a $\mathrm{Cu}-\mathrm{K} \alpha$ source $(0.15418 \mathrm{~nm})$ in the standard Bragg-Brentano geometry. The accelerating voltage in both cases was $40 \mathrm{kV}$ and the tube current $40 \mathrm{~mA}$. The time per step was $5 \mathrm{~s}$. For the XRD the step size was $0.05^{\circ}$ and the angle of incidence $(\Omega)$ was $1^{\circ}$. For the XRR the step size was $0.005^{\circ}$. In both cases the beams were collimated and filtered.

Reflection and transmission spectra of the thin films were acquired using a Perkin Elmer 950 Lambda UV/Vis/NIR at an angle of incidence of $8^{\circ}$ and a Varian Cary 5E UV/Vis/NIR at incident angle of $0^{\circ}$, respectively. Estimates of the dielectric function of the alloy samples were extracted with WVASE32 (product of J A Woollam Co., Inc. of Lincoln, NE 68508, USA). The optical constants were obtained by fitting Lorentz oscillator models to the transmission and reflection spectra, which ensured the KramersKronig consistency.

\section{Results and Discussion}

\subsection{Metastable $(\mathrm{Au}, \mathrm{Ni})$ alloys}

Fig. 2 shows the total electronic DOS and the partial DOS for the Ni $d$-states for each alloy modeled. The DOS for pure gold is dominated by the $d$-band and the appearance of the transition metal dopant states (of Ni) as a VBS just below the Fermi level is clearly observed. The data are in qualitative agreement with the experimental valence band spectra for $\mathrm{Au}_{(1-x)} \mathrm{Ni}_{x}$ and with much earlier calculations .

The theoretical reflectivity spectra of Fig. 3a show that, as the nickel concentration increases, the reflectivity at long wavelengths should be attenuated relative to that of pure gold and the absorption edge should shift to shorter wavelengths (arrow). The calculated reflectivity of the $\mathrm{Au}_{0.875} \mathrm{Ni}_{0.125}$ compares very favorably to the measured reflectivity of a range of actual $\mathrm{Au}_{0.85} \mathrm{Ni}_{0.15}$ samples of different thickness, 
especially when referenced to pure $\mathrm{Au}$, as shown in Fig. 3b. The changes in reflectivity can be attributed to the additional interband transitions from the VBS. At lower concentrations of Ni the theoretical reflectivity is intermediate in shape but the trend is not exactly monotonic indicating that precise details of the interband transitions are probably important. Once the alloy concentration increases to 25 at $\%$ the Ni states appear to introduce an additional free-electron like contribution to the reflectivity and it evolves towards the reflectivity of pure $\mathrm{Ni}$. At wavelengths shorter than the band edge, $\mathrm{Au}_{0.875} \mathrm{Ni}_{0.125}$ and $\mathrm{Au}_{0.75} \mathrm{Ni}_{0.25}$ have a reflectance greater than that of pure $\mathrm{Au}$, i.e. their interband transitions have been attenuated. This is consistent with the decrease in the $\mathrm{Au} d$-band DOS. In contrast the 3.125at $\% \mathrm{Ni}$ alloy has less reflectance in this region i.e. it has stronger interband transitions than pure Au. However, overall pure Au has a greater differential in reflectivity above and below the band edge and hence a stronger color. Reduction of this differential is hence associated with bleaching of the color.

Variation of the thickness of films of fixed stoichiometry provides another way to control spectral properties. The experimental $\mathrm{Au}_{0.85} \mathrm{Ni}_{0.15}$ films became more reflective (and whiter) as they became thicker. This is because less light was lost by transmittance through the thicker coatings. Despite this variation in reflectance, the dielectric functions of these experimental alloy films were tightly clustered, Fig.4, indicating that the extracted values were a true material property. However, it is clear that the alloy films would exhibit greater optical 'loss' than either the pure elements $\mathrm{Au}$ and $\mathrm{Ni}$, or the computed metastable alloys.

\subsection{Thin film stacks of the elements}

The other route to controlling the spectral-selectivity of the reflectance is to lay down separate multilayers of $\mathrm{Au}$ and $\mathrm{Ni}$. In principle many alternating layers can be deposited but in practice the roughness of the interlayer interfaces increases with each additional layer, Fig. 5. This complicates analysis using standard thin film software. Therefore, in the present paper we restrict our detailed analysis to tri-layer stacks. 
The fcc structure of Au within a three layer sample was evident in laboratory XRD, Fig. 6a, although the diffraction peaks were relatively broad and a degree of strain in the film was required in order to fit the data. Clearly, the as-deposited material, while partially crystallized, is nevertheless relatively disordered and strained. This is an important point as it is reasonable to expect that such microstructures would experience greater electron scattering and hence optical loss than fully crystallized material. In Fig. 6b we show X-ray reflectometry (XRR) data for this sample and in Fig. 6c we show its measured and simulated reflectance and transmittance curves. There is a broad transmittance peak at about $500 \mathrm{~nm}$. The simulated reflectance curves show slight inflections and detail that are lacking in the measured ones. This is due to the actual sputtered layers of Au and Ni not having perfectly planar and smooth interfaces. Some roughening of the interfaces would result in the attenuation of the fine features predicted by the simulation. Fitting of a X ray scattering density model to the XRR data indicated a stack of approximately $\mathrm{Au}(7 \mathrm{~nm})-\mathrm{Ni}(4 \mathrm{~nm})-\mathrm{Au}(7 \mathrm{~nm})$ whereas fitting of an optical model to the reflectance and transmittance indicated a stack of approximately $\mathrm{Au}(7 \mathrm{~nm})-\mathrm{Ni}(5 \mathrm{~nm})-\mathrm{Au}(7 \mathrm{~nm})$. Note that the simulations (X-ray or optical) used standard material data for the bulk elements. An interfacial roughness of $1 \mathrm{~nm}$ was applied for the XRR fit but, as mentioned, the optical simulations assumed perfectly smooth layers.

We then used the OpenFilters program to make an extended numerical exploration of the optical properties available from such tri-layer systems. In this scheme we assumed a perfectly smooth tri-layer $\mathrm{Au}-$ $\mathrm{Ni}-\mathrm{Au}$ coating with the thickness of the two Au films made identical. The color gamut possible in reflection from this system is shown in Fig. 7. CIE L*a*b* color coordinates have been mapped to their approximate RGB colors in the diagram. A wide range of possibilities can be achieved. As the thickness of the Au layer increases beyond $50 \mathrm{~nm}$ the colors become similar to those of pure gold. Conversely, the colors become similar to those of elemental $\mathrm{Ni}$ as the thickness of the Ni layer increases beyond $10 \mathrm{~nm}$ and the $\mathrm{Au}$ decreases below $4 \mathrm{~nm}$. However, the colors for intermediate structures are not merely a linear interpolation between those of the pure elements. The measured colors of the $\mathrm{Au}_{0.85} \mathrm{Ni}_{0.15}$ alloy samples and the tri-layer sample are superimposed on the data. The former can be seen to be spread across the range of predicted colors depending on their thickness, while the perceived color of the latter is displaced (red arrow) towards red due to the bulk dielectric functions used to calculate the color gamut being slightly different to the actual 
dielectric functions of the nanoscale Au and Ni layers. The displacement is due to the color perceived by the eye being exceedingly sensitive to small variations in the mid-visible region, notwithstanding the good agreement between data and model in Fig. 6c. If required, the tri-layer simulations could be improved further if measured dielectric functions of nanoscale elemental films were used, rather than the standard properties of annealed bulk elements.

\section{Conclusion}

We have used a combination of calculation and experiment to demonstrate two different techniques to tune the spectral properties of $\mathrm{Au}-\mathrm{Ni}$ thin films. In the first method, the dielectric function of the alloy making up the film can be tuned by control of the electronic configuration of a metastable solid solution. The results of the $a b$ initio calculations are broadly similar to those of the experimental films thereby validating the approach. In the second approach, the optical properties of a composite Au-Ni thin film stack were tuned by varying the number and thickness of the layers. Once again, the calculated reflectance spectra and colors were similar to the measured ones. Therefore, to a first approximation, the two approaches (metastable solid solution or thin film stack) are interchangeable, and either offers a convenient means to interpolate the optical properties of these films between those of $\mathrm{Au}$ and $\mathrm{Ni}$.

\section{Acknowledgements}

The authors thank the Australian Research Council and the Australian Nuclear Science and Technology Organisation for support, and Mr Guilhem Capdeboscq for assisting with measurements. 


\section{References}

[1] M. Blaber, M.J. Ford, M.B. Cortie, in: C. Corti, R. Holliday (Eds.), Gold. Science and Applications, Taylor and Francis, London, 2009, p. 13.

[2] G. Normandeau, Gold Bull. 25/3 (1992) 94.

[3] T. Shiraishi, J. Geis-Gerstorfer, Gold Bull. 39/1 (2006) 9.

[4] T. Shiraishi, K. Hisatsune, Y. Tanaka, E. Miura, Y. Takuma, Gold Bull. 34/4 (2001) 129.

[5] J.L. Murray, Alloy Phase Diagrams, ASM Materials International, Materials Park, Ohio, 2002.

[6] M. Bassett, D. Beaglehole, J. Phys. F: Metal Phys. 6/6 (1976) 1211.

[7] D. Beaglehole, T.J. Hendrickson, Phys. Rev. Lett. 22/4 (1969) 133.

[8] G. Meng, R. Claesseny, F. Reinert, R. Zimmermann, P. Steiner, S. Hüfner, J. Phys.: Condens. Matter 8 (1996) 5569.

[9] P. Blaha, K. Schwarz, G.K.H. Madsen, D. Kvasnicka, J. Luitz, WIEN2k, An Augmented Plane Wave + Local Orbitals Program for Calculating Crystal Properties Techn. Universität Wien, Vienna, Austria, 2001.

[10] C. Ambrosch-Draxl, J.O. Sofo, Comp. Phys. Comm. 175 (2006) 1.

[11] S. Larouche, L. Martinu, Appl. Optics 47/13 (2008) C219.

[12] J.H. Weaver, H.P.R. Frederikse, in: D.R. Lide (Ed.), CRC Handbook of Chemistry and Physics, CRC Press, Boca Raton, 2001, p. 133.

[13] P. Weinberger, J. Phys. F: Met. Phys. 13 (1983) 625.

[14] J.H. Wang, X.G. Lu, B. Sundman, X.P. Su, Calphad-Computer Coupling of Phase Diagrams and Thermochemistry 29/4 (2005) 263. 
Figures

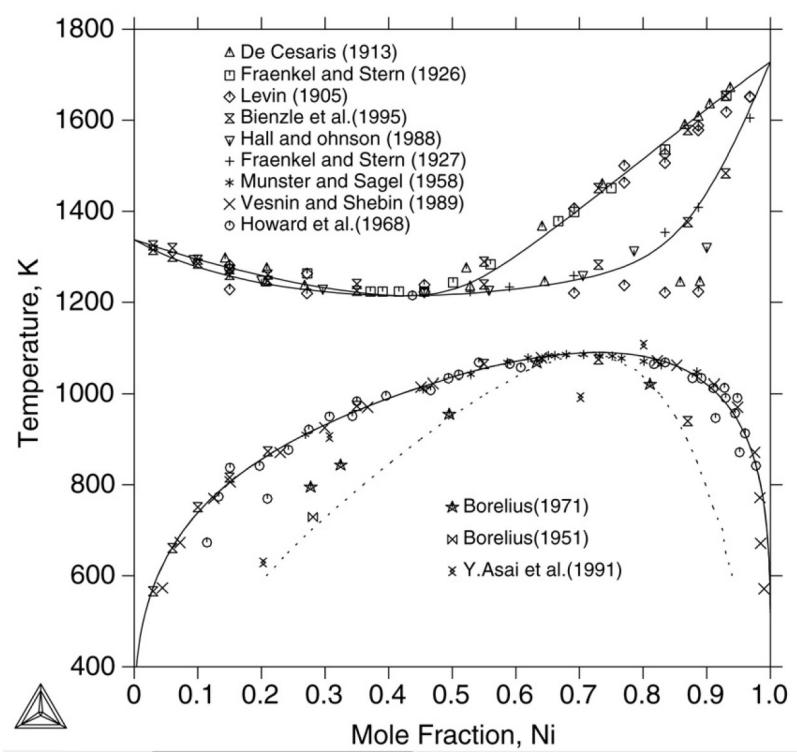

Fig. 1. Equilibrium phase diagram of the Au-Ni system. Reproduced with permission from J. H. Wang, X. G. Lu, B. Sundman, X. P. Su, Calphad, 2005, 29, 263. Copyright 2005, Elsevier . Note the negligible solubility of $\mathrm{Au}$ in $\mathrm{Ni}$, or vice versa, at temperatures below about $400 \mathrm{~K}$. The dotted line shows the estimated limit of the spinodal decomposition. 


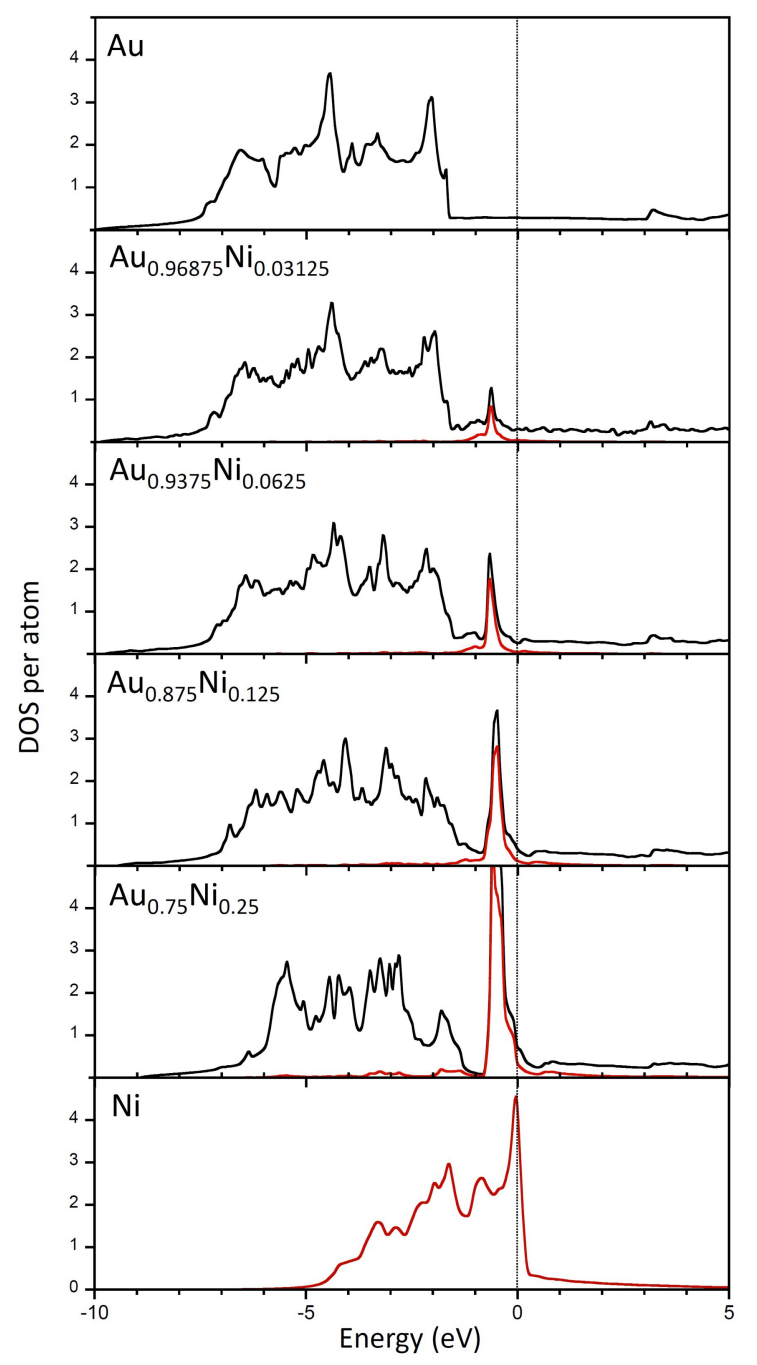

Fig. 2. Calculated total DOS (black) and Ni $d$-states (red) for $\mathrm{Au}_{(1-x)} \mathrm{Ni}_{x}$ alloys. 
(a)
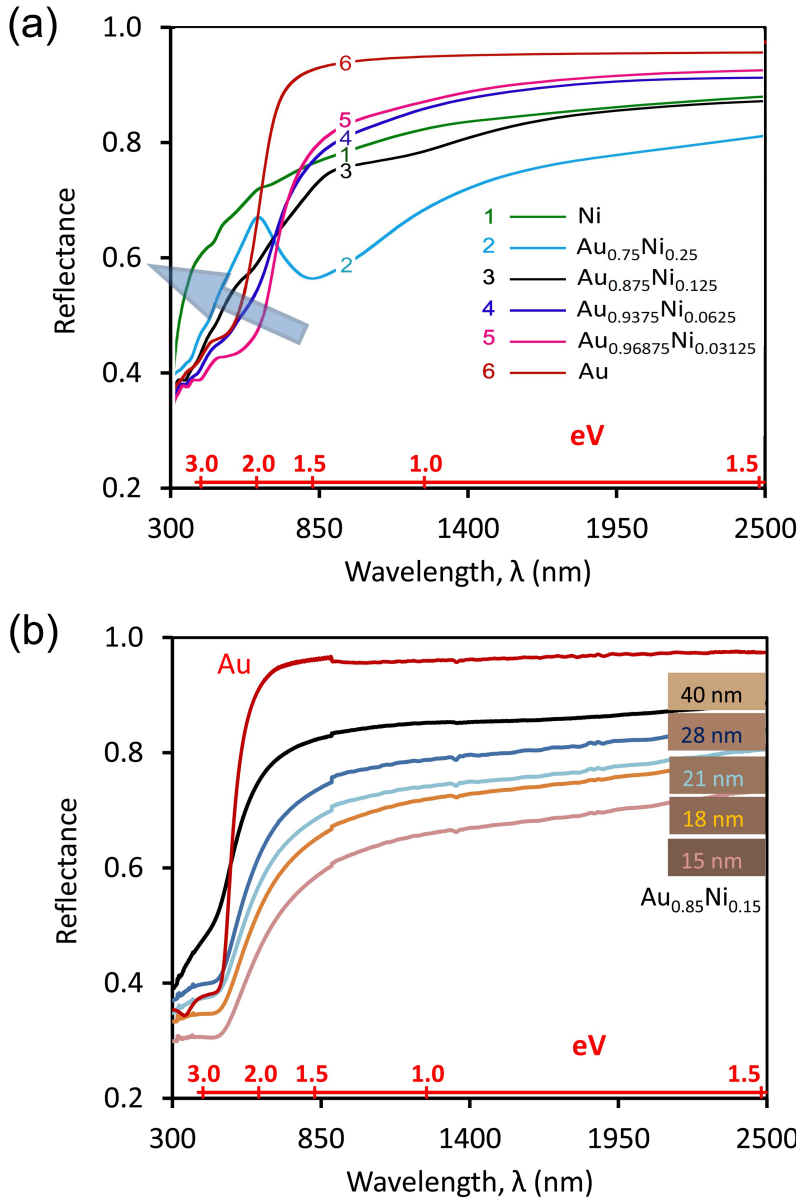

Fig. 3. (a) Reflectivity calculated for a series of bulk $\mathrm{Au}_{1-x} \mathrm{Ni}_{x}$ alloys using DFT. (b) Reflectance of experimental $\mathrm{Au}_{0.85} \mathrm{Ni}_{0.15}$ thin films of various thicknesses. The reflectance of a thick $(100 \mathrm{~nm}) \mathrm{Au}$ layer is shown for purpose of comparison. 

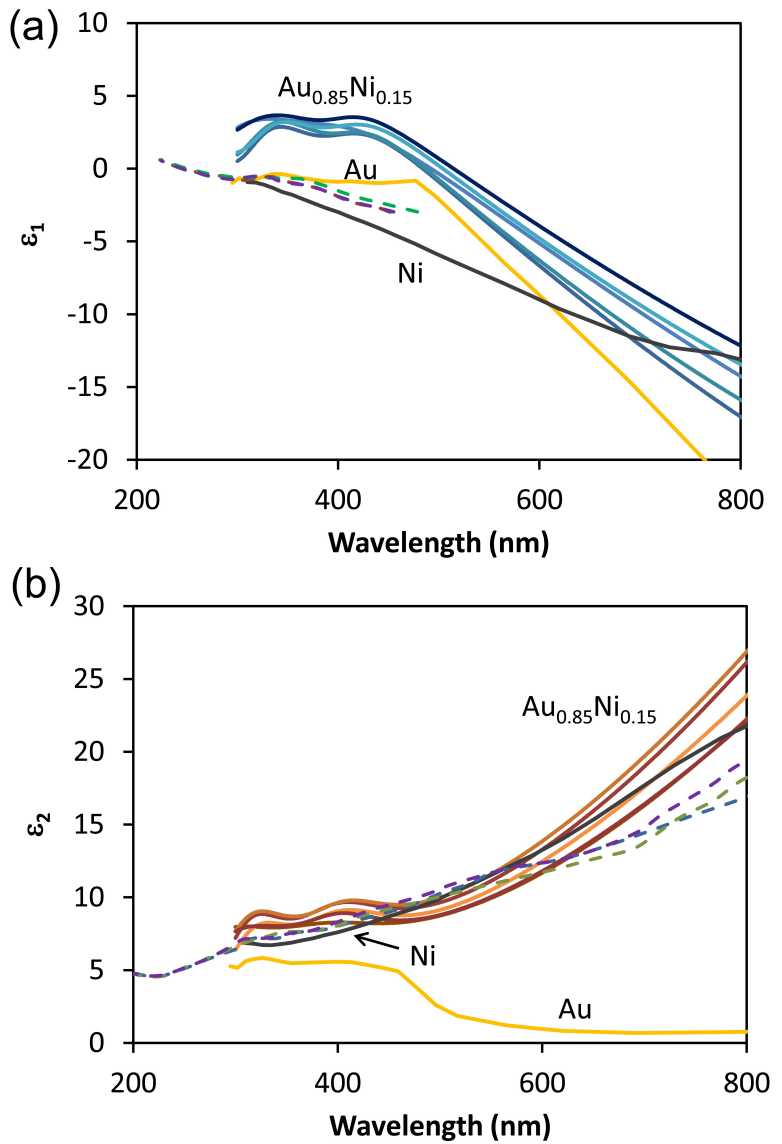

Fig. 4. Dielectric functions of $\mathrm{Au}_{0.85} \mathrm{Ni}_{0.15}$ alloy films of varying thicknesses, above $\varepsilon_{1}$ as blue lines, below $\varepsilon_{2}$ as brown lines. The dielectric functions of pure, bulk Au and $\mathrm{Ni}$ (solid yellow and grey lines respectively) and the calculated dielectric function of $\mathrm{Au}_{0.875} \mathrm{Ni}_{0.125}$ (dashed lines, various unit cells) are shown for comparison. As discussed in the main text, the alloy films are metastable and contain a high content of structural defects which causes them to have optical 'loss' than the pure elements or the simulated alloys.

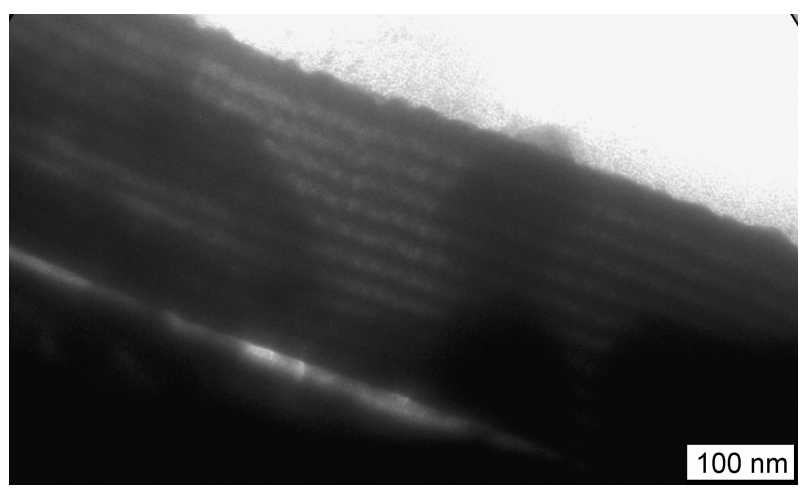

Fig. 5. Transmission electron microscope cross-section taken through a twenty-layer stack of alternating $\mathrm{Au}$ (dark) and $\mathrm{Ni}$ (light) produced by magnetron sputtering. Although the layers are conformal, there is clearly a build-up of interlayer roughness as successive deposits are made. 
(a)

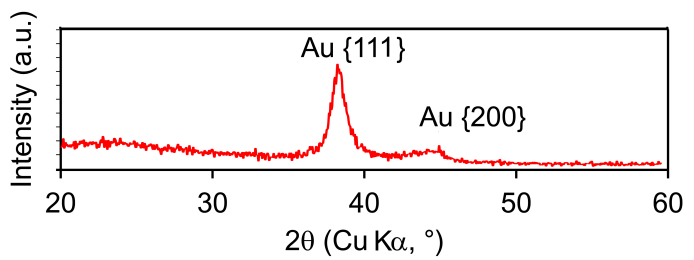

(b)

$2 \theta\left(\mathrm{CuK} \alpha,{ }^{\circ}\right)$

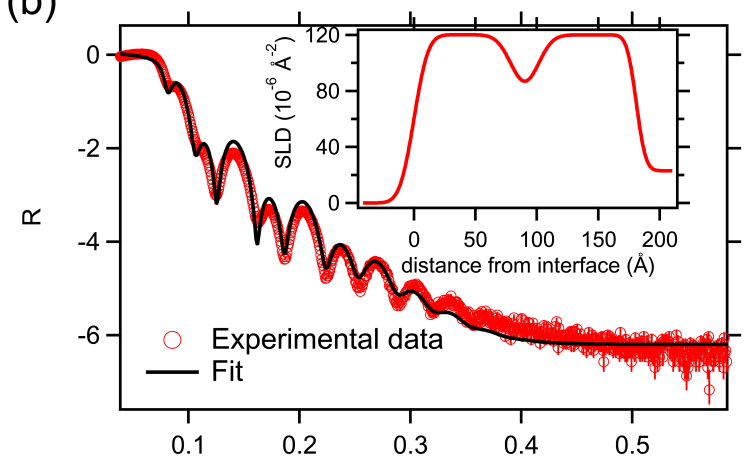

(c)

$\mathrm{Q}\left(\AA^{-1}\right)$

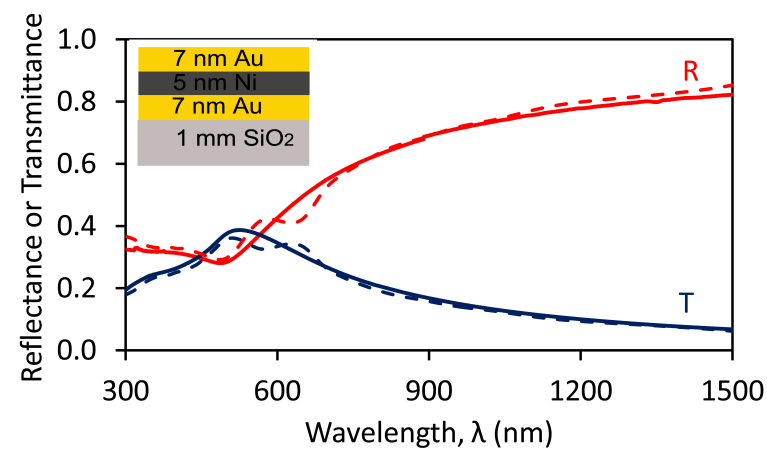

Fig. 6. (a) Portion of XRD pattern (glancing angle) of tri-layer coating, showing peaks due to gold only. (b) XRR data with superimposed fitted model showing tri-layer structure. (c) Optical properties of the tri-layer sample, with simulation (dashed lines) obtained using literature dielectric data and the thicknesses in (b).

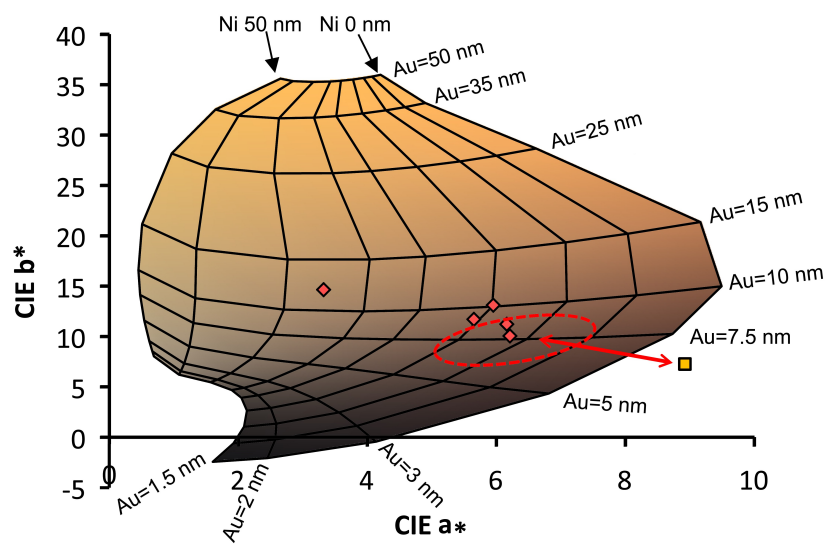

Fig. 7. Computed reflective CIE $L^{*} a * b^{*}$ color gamut of Au-Ni-Au tri-layer on $1 \mathrm{~mm}$ clear $\mathrm{SiO}_{2}$. Also shown are the measured coordinates of the series of $\mathrm{Au}_{0.85} \mathrm{Ni}_{0.15}$ alloy samples (diamond symbols), an experimental Au-Ni-Au tri-layer (square), and the locus (dashed red oval) of calculated colors of ideal tri-layers of similar dimensions to the experimental one. 